American Journal of

Health, Medicine and Nursing Practice (AJHMN)

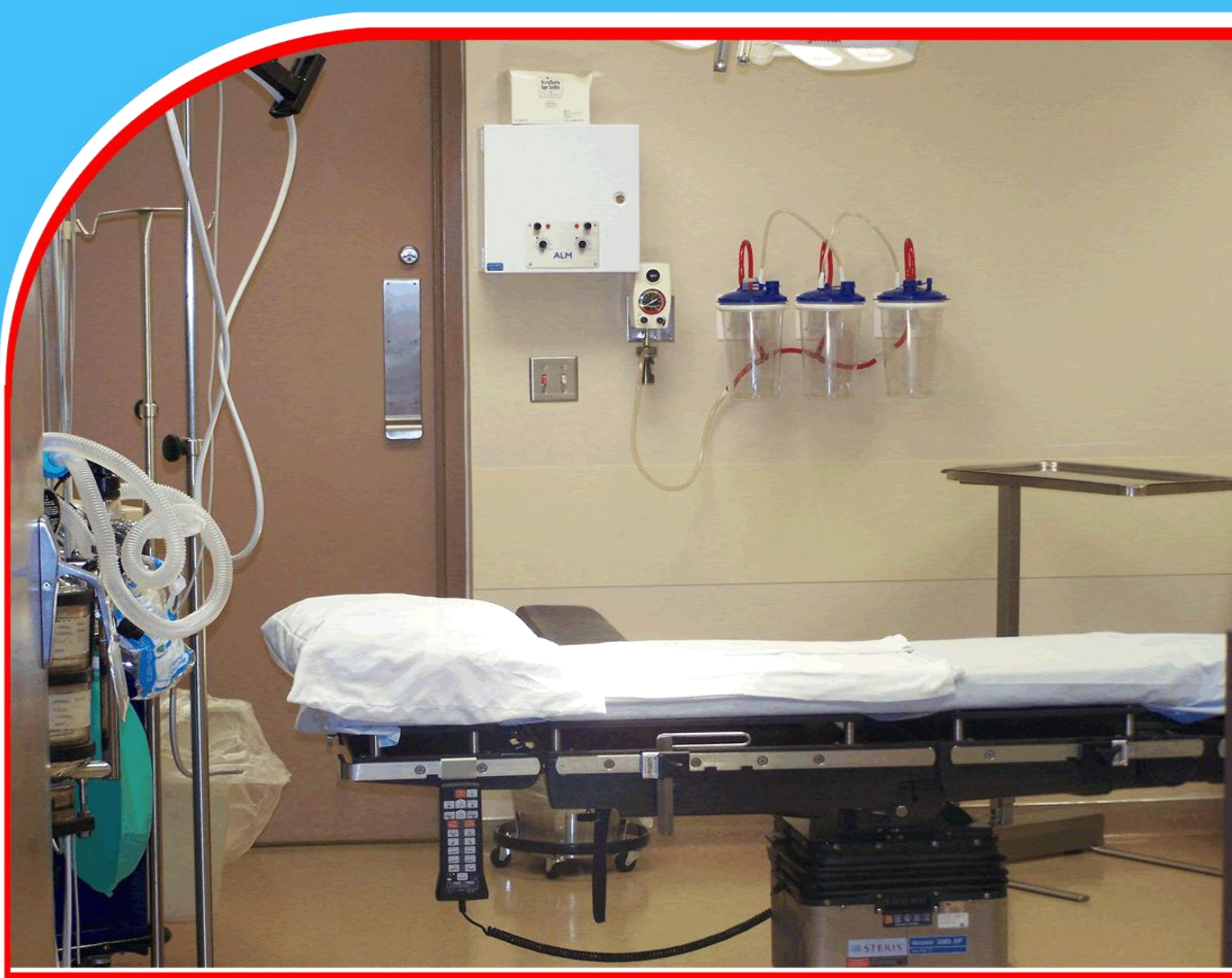

Result of a standardized management protocol for chronic orchialgia and a suggested algorithm incorporating spermatic cord block, tender point block, microscopic vericocelectomy, and microscopic sub inguinal denervation. Mir Abid Jan, Muhammad Waqas, Naveed, Qudrat, Ishan Ullah Khan, Ikram, and Samiullah Opal

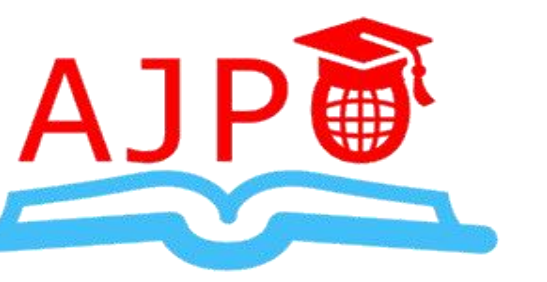




\title{
Result of a standardized management protocol for chronic orchialgia and a suggested algorithm incorporating spermatic cord block, tender point block, microscopic vericocelectomy, and microscopic sub inguinal denervation.
}

\author{
${ }^{1}$ Mir Abid Jan, ${ }^{2}$ Muhammad Waqas, ${ }^{3}$ Naveed, ${ }^{4}$ Qudrat, ${ }^{5}$ Ishan Ullah Khan, ${ }^{6}$ Ikram, \\ ${ }^{7}$ Samiullah Opal \\ ${ }^{1}$ HOD Assistant professor Andrologist and Urologist Institute Of Kidney Disease Peshawar \\ Pakistan \\ ${ }^{2}$ Senior Registrar kidney center Bahawalpur, Pakistan \\ 3,4,5,6,7 Medical Officer Institute of kidney disease, Peshawar, Pakistan \\ Corresponding Authors: Ishan Khan, Naveed \\ Emails: Ihsandru@gmail.com, khan199234@gmail.com
}

\begin{abstract}
Purpose: We investigated Patients presenting with chronic orchialgia at Andrology in institute of kidney and diseases Peshawar, from 2003 August up to when were included.

Materials and Methods: A thorough history and physical examination was undertaken including description of pain by the patient in terms of site, severity, radiation and associated pain. Extensive workup, directed by history and phsical examination, was done to rule out reversible causes of orchalgia. All patients had urinalysis, culture and ultrasound scrotum with color Doppler. Further investigations like semen analysis, culture and hormonal workup were done if indicated. The intensity of the pain was noted according to visual analogue scale. Patients were subdivided into three groups as mild pain (group A, pain score $=1$ - 3), moderate pain (group B, pain score $=4$ - 6) and severe pain, (group C, pain score $=7$ - 10). Site of pain and radiation/association to any other region was recorded.

Finding: Results of the study indicated that 92 patients reported at institute of kidney diseases Peshawar with chronic orchialgia had their mean age at 37+/-4years. Five patients lost to follow so 92 were included in final analysis (table 01). Pain was partially relieved in 14 patients and not relieved in another 9 patients which is almost $76 \%$ of total patients. These non-responders were compared with the remaining in which pain was completely relieved. There was no difference in etiology among responders and non-responders, however pain severity was more in nonresponders at initial presentation (table 2).

Conclusions: Patients with pelvic floor muscle spasm are more likely to experience treatment failure following microscopic subinguinal spermatic cord denervation for chronic scrotal content pain, even with a favorable response to spermatic cord block. A history relating to pelvic floor muscle spasm should be taken for all patients presenting with chronic orchialgia or chronic scrotal content pain, and digital rectal exam should be performed if the history is suggestive. If underlying pelvic floor dysfunction exists, pelvic floor physical therapy can be offered to patients prior to spermatic cord denervation. History of prior vasectomy, epididymectomy, prior inguinal or scrotal surgery or other patient demographic factors were not associated with treatment failure.
\end{abstract}

Keyword: Chronic orchialgia, spermatic cord block, vericocelectomy, subinguinal 
American Journal of Health, Medicine and Nursing Practice

ISSN 2520-4017 (Online)

Vol.7, Issue 3, pp $1-7,2022$

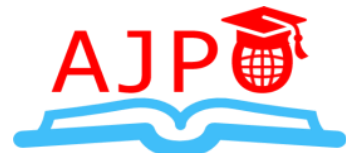

WWW.ajpojournals.org

\section{INTRODUCTION}

Chronic orchialgia is defined as " intermittent or constant, unilateral or bilateral testicular pain, 3 months or longer in duration that significant significantly interferes with the daily activities of the patient so as to prompt him to medical intervene intervention"1 It has been suggested that in socalled chronic orchalgia, Pam may not be limited to tech nididymis. ${ }^{2-4}$ vas deferens, or adjacent Para testicular structures may also be painful Therefore such pain may be more on scrotal content pain 21 The pathogenesis of this condition is poorly understood and the effect of underlying conditions e.g. varicocele and underlying conditions e.g. varicocele and post vasectomy sperm granuloma is unpredictable. Chronic orchalgia is agonizing. ${ }^{4-6}$ Chronic orchalgia is agonizing and stressful for the patients and has significant impact on their quality of life in severe cases patients the touch of routine dress garments. Treatment of these patients is largely empirical. First line tar patients is largely empirical. First line treatment usually consist of analgesics combined with pain modulators like tricycle antidepressants and modulators like tricycle antidepressants and Gabapentin ${ }^{6-9}$. Spermatic cord block are used both for therapeutic relief of pain a pot for therapeutic relief of pain and to assess response and decide for microsurgical enervation ${ }^{10}$.

There is no consensus on when to proceed for surgical therapy Surgical management is usually reserved for the patients in whom medical treatment is not effective or when an underlying disease is identified which is amenable to surgical intervention. ${ }^{11-14}$ Surgical options include epididymectomy and vasectomy reversal in patients $\mathrm{w}$ post vasectomy pain; and Microsurgical enervation of spermatic cord (MDS) ${ }^{15-16}$ In patients with chronic orchialgia and varicocele, ${ }^{17-18}$ microsurgical varicocelectomy is usually offered, but complete response rates vary from 53 to $91 \%$ $[9,10)$. Owing to uncommon presentation and lack of standard protocols, ${ }^{19-20}$ treatment of chronic orchalgia is largely empirical based upon personal experiences and available resources. We present short term results of patients presenting with chronic orchalgia in our andrology clinic, ${ }^{21}$ managed using a standardized protocol. We also suggest an algorithm based upon our results.

\section{METHODS}

Conservative treatment options are presented and then targeted surgical interventions that the urologist may perform are then presented in a structured algorithm format. Many of these patients may obtain a significant reduction in pain with some of these treatments. Patients presenting with chronic orchialgia at Andrology in institute of kidney and diseases Peshawar, from 2003 August up to when were included. A thorough history and physical examination was undertaken including description of pain by the patient in terms of site, severity, radiation and associated pain. Extensive workup, directed by history and phsical examination, was done to rule out reversible causes of orchalgia. All patients had urinalysis, culture and ultrasound scrotum with color Doppler. Further investigations like semen analysis, culture and hormonal workup were done if indicated. The intensity of the pain was noted according to visual analogue scale. Patients were subdivided into three groups as mild pain (group A, pain score $=1$ - 3), moderate pain (group B, pain score $=4$ - 6) and severe pain, (group C, pain score $=7$ - 10). Site of pain and radiation/association to any other region was recorded. Patients were managed for chronic orchialgia according to a standardized protocol and prospectively followed for pain resolution categorized as complete relief, partial relief and no relief. 
Management protocol for orchalgia: Patients were initially managed by oral analgesics with or without antiinflammtory medicines. Antibiotics were prescribed where indicated. Non-responders were offered spermatic cord block. A combination of Bupivicaine $3 \mathrm{ml}+$ Triamcinolone $10 \mathrm{mg}$ was used for injections. If tender points other than scrotum identified during examination, high ilio-inguinal nerve block or paravertebral block for tender points were administered. Microsurgical varicocelectomy was offered to patients who had varicocele on clinical examination and had more than $2.5 \mathrm{~mm}$ diameter varicocele veins on color Doppler ultrasonography. Microsurgical denervation of spermatic cord was offered if pain was not responding to oral medications but temporarily relieved with spermatic cord block as suggested by Benson (5). Microsurgical denervation was also offered to patients undergoing microsurgical varicocelectomy if they responded to spermatic cord block. All surgical procedures were carried out by a single Fellowship trained surgeon using standard technique. Patients were referred to pain clinic for further management if pain was not relieved at all.

\section{Table 01: Patients Demographics}

\section{DEMOGRAPHICS}

No.patients

No. surgeries included

Mean age (years, SD, range)

Right

Lacterality $-(\%)$

Prior vasectomy, n (\%)

Prior epididymectomy, n (\%)

Other prior inguinal/scrotal surgery, $\mathrm{n}$

Pelvic floor muscle spasm, n(\%)

Median time with orchialgia ( months, Q1-Q3)

Mean BMI $\left(\mathrm{Kg} / \mathrm{m}^{2}, \mathrm{SD}\right.$, range

Resolution of pain, $\mathrm{n}(\%)$

\section{VALUE}

92

114

$37+4(13.2: 20-88)$

$22(22 \%)$

$32(\%)$

$9(8.8 \%)$

$50(49 \%)$

$19(19 \%)$

24(12-60)

$29.4(6.0: 18.1-47.1)$

$92(76 \%)$ 
American Journal of Health, Medicine and Nursing Practice

ISSN 2520-4017 (Online)

Vol.7, Issue 3, pp $1-7,2022$

www.ajpojournals.org

Table 02: Predictors of MSCD Failure

\begin{tabular}{lccc}
\hline No of patients 92 & Odds ratio & $95 \%$ CI & P value \\
\hline Age (per year) & 0.98 & $0.95-1.02$ & 036 \\
BM1 (per kem2) & 0.96 & $0.91-1.06$ & 0.70 \\
Months of Pain (per month) & 1.00 & $0.99-1.00$ & 0.48 \\
Side (ft vs. L) & 1.18 & $0.46-3.00$ & 0.74 \\
Prior vasectomy & 1.45 & $0.47-4.50$ & 0.52 \\
Prior epididymectomy & 0.60 & $0.09-3.90$ & 0.60 \\
Other prior inguinalserotal surgery & 0.99 & $038-2.66$ & 0.99 \\
Presence of pelvic floor muscle spasm & 3.95 & $1.27-1226$ & 0.02 \\
\hline
\end{tabular}

\section{RESULTS}

From August 2003 to April 2011, 92 patients reported at institute of kidney diseases Peshawar with chronic orchialgia. Their mean age was 37+/-4years. Five patients lost to follow so 92 were included in final analysis (table 1). Pain was partially relieved in 14 patients and not relieved in another 9 patients which is almost $76 \%$ of total patients. These non-responders were compared with the remaining in which pain was completely relieved. There was no difference in etiology among responders and non-responders, however pain severity was more in non-responders at initial presentation (table 2). Reaming patients were found to have additional pain sites (APS) besides inguinoscrotal region. These APS included ipsilateral or contralateral thighs; iliac, supraumblical and lumbo-sacral regions. Relevant radiological investigations for these additional pain sites and scrotal color Doppler ultrasound were found un-remarkable. Pain in these APS occurred in ipsilateral or sometimes contralateral testis and did occur occasionally independent of scrotal pain in 5 patients. Oral analgesics provided no relieve in pain, however spermatic cord block not only relieved their scrotal pain, but also provided a durable relief to these APS at 6month follow-up.

\section{DISCUSSION}

There is a great variability in the management of chronic Orchialgia. Various medical as well as surgical options have been described. This variability stems from our incomplete understanding of pathophysiology this uncommon condition. Various new treatment options like Electro puncture in combination with amitryptaline, multiphoton microscopy and ablation of selective nerves, pulsed radio frequency denervation of spermatic cord etc. have been proposed for management of chronic orchialgia. The complexity of this disorder demands a methodical management plan and resources which are usually only available at dedicated centers. We suggest a management algorithm which takes into account the underlying etiology and response to spermatic cord block as suggested by Benson (5). Microsurgical denervation (MSD) should be offered if the patient has 
American Journal of Health, Medicine and Nursing Practice ISSN 2520-4017 (Online)

Vol.7, Issue 3, pp $1-7,2022$

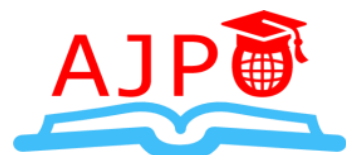

www.ajpojournals.org

responded to spermatic cord block. This MSD can be combined with microscopic varicocelectomy if the patient also has clinical varicocele (Figure 1) Varicocele is commonly linked with chronic orchialgia and its treatment relieves pain in most, but not all of the patients.

Also many patients with varicocele do not have chronic orchalgia and vice versa suggesting other factors may have a role in pathogenesis. In our study regardless of etiology the response to treatment was not different in varicocele as well as in non-varicocele patients. This suggests that our methodical approach to chronic orchalgia remains valid irrespective of underlying etiology and can help physicians in making a decision. Levine has suggested that this pain should be named as chronic spermatic cord content pain due to its potential to radiate. In our experience the pain radiates even farther than the scrotal contents to as far as ipsilateral or contralateral, thigh, inguinal and suprapubic region. Interestingly it was found that this referred pain gets relieved by spermatic cord block which suggests that it is linked with spermatic cord contents. Further studies and investigations (nerve conduction studies or EEG) is required to understand the exact mechanism of this un-explored phenomenon.

\section{CONCLUSION}

Our Finding that 92 patients reported at institute of kidney diseases Peshawar with chronic orchialgia. Their mean age was 37+/-4years. Five patients lost to follow so 92 were included in final analysis (table 01). Pain was partially relieved in 14 patients and not relieved in another 9 patients.its almost $\mathbf{7 6 \%}$ of total patients. These non-responders were compared with the remaining in which pain was completely relieved. There was no difference in etiology among responders and non-responders, however pain severity was more in non-responders at initial presentation (table 2).

\section{REFERENCES}

1. Benson JS, Abern MR, Larsen S, et al. Does a Positive Response to Spermatic Cord Block Predict Response to Microdenervation of the Spermatic Cord for Chronic Scrotal Content Pain? J Sex Med 2013;10:876-82. 10.1111/j.1743-6109.2012.02937.x [PubMed] [CrossRef] [Google Scholar]

2. Strom KH, Levine LA. Microsurgical Denervation of the Spermatic Cord for Chronic Orchialgia: Long-Term Results From a Single Center. J Urol 2008;180:949-53. 10.1016/j.juro.2008.05.018 [PubMed] [CrossRef] [Google Scholar]

3. Devine CJ, Schellhammer PF. The use of microsurgical denervation of the spermatic cord for orchialgia. Trans Am Assoc Genitourin Surg 1978;70:149-51. [PubMed] [Google Scholar]

4. Marconi M, Palma C, Troncoso P, et al. Microsurgical Spermatic Cord Denervation as a Treatment for Chronic Scrotal Content Pain: A Multicenter Open Label Trial. $J$ Urol 2015;194:1323-7. 10.1016/j.juro.2015.05.081 [PubMed] [CrossRef] [Google Scholar]

5. Calixte N, Tojuola B, Kartal I, et al. Targeted Robotic Assisted Microsurgical Denervation of the Spermatic Cord for the Treatment of Chronic Orchialgia or Groin Pain: A Single Center, Large Series Review. J Urol 2018;199:1015-22. 10.1016/j.juro.2017.10.030 [PubMed] [CrossRef] [Google Scholar] 
American Journal of Health, Medicine and Nursing Practice

ISSN 2520-4017 (Online)

Vol.7, Issue 3, pp 1-7, 2022

www.ajpojournals.org

6. Shiraishi K, Tabara M, Matsuyama H. High Inguinal Microsurgical Denervation of the Spermatic Cord for Chronic Scrotal Content Pain: A Novel Approach for Adult and Pediatric Patients. Urology 2019;131:144-9. 10.1016/j.urology.2019.05.013 [PubMed] [CrossRef] [Google Scholar]

7. Heidenreich A, Olbert P, Engelmann UH. Management of Chronic Testalgia by Microsurgical Testicular Denervation. Eur Urol 2002;41:392-7. 10.1016/S0302-2838(02)00023-4 [PubMed] [CrossRef] [Google Scholar]

8. Parekattil SJ, Gudeloglu A, Brahmbhatt JV, et al. Trifecta Nerve Complex: Potential Anatomical Basis for Microsurgical Denervation of the Spermatic Cord for Chronic Orchialgia. $J$ Urol 2013;190:265-70. 10.1016/j.juro.2013.01.045 [PubMed] [CrossRef] [Google Scholar]

9. Kavoussi PK. Validation of targeted microsurgical spermatic cord denervation: comparison of outcomes to traditional complete microsurgical spermatic cord denervation. Asian $J$ Androl 2019;21:319-23. 10.4103/aja.aja_87_18

10. Wagenlehner FME, van Till JWO, Magri V, et al. National Institutes of Health Chronic Prostatitis Symptom Index (NIH-CPSI) Symptom Evaluation in Multinational Cohorts of Patients with Chronic Prostatitis/Chronic Pelvic Pain Syndrome. Eur Urol 2013;63:953-9. 10.1016/j.eururo.2012.10.042 [PubMed] [CrossRef] [Google Scholar]

11. Shoskes DA, Calixte N, Tadros N, et al. Validation of the Chronic Orchialgia Symptom Index for Men With Chronic Orchialgia/Chronic Scrotal Contents Pain. Urology 2018;119:3943. 10.1016/j.urology.2018.05.030 [PubMed] [CrossRef] [Google Scholar]

12. Rotshenker S. Wallerian degeneration: the innate-immune response to traumatic nerve injury. J Neuroinflammation 2011;8:109. 10.1186/1742-2094-8-109 [PMC free article] [PubMed] [CrossRef] [Google Scholar]

13. Dubový P. Wallerian degeneration and peripheral nerve conditions for both axonal regeneration and neuropathic pain induction. Ann Anat 2011;193:267-75. 10.1016/j.aanat.2011.02.011 [PubMed] [CrossRef] [Google Scholar]

14. Üçeyler N, Sommer C. Wallerian degeneration and neuropathic pain. Drug Discov Today Dis Mech 2006;3:351-6. 10.1016/j.ddmec.2006.09.004 [CrossRef] [Google Scholar]

15. Oka S, Shiraishi K, Matsuyama H. Microsurgical Anatomy of the Spermatic Cord and Spermatic Fascia: Distribution of Lymphatics, and Sensory and Autonomic Nerves. $J$ Urol 2016;195:1841-7. 10.1016/j.juro.2015.11.041 [PubMed] [CrossRef] [Google Scholar]

16. Shah JP, Danoff JV, Desai MJ, et al. Biochemicals associated with pain and inflammation are elevated in sites near to and remote from active myofascial trigger points. Arch Phys Med Rehabil 2008;89:16-23. 10.1016/j.apmr.2007.10.018 [PubMed] [CrossRef] [Google Scholar]

17. Moldwin RM, Fariello JY. Myofascial Trigger Points of the Pelvic Floor: Associations with Urological Pain Syndromes and Treatment Strategies Including Injection Therapy. Curr Urol Rep 2013;14:409-17. 10.1007/s11934-013-0360-7 [PubMed] [CrossRef] [Google Scholar] 
American Journal of Health, Medicine and Nursing Practice

ISSN 2520-4017 (Online)

Vol.7, Issue 3, pp $1-7,2022$

www.ajpojournals.org

18. Planken E, Voorham-van der Zalm PJ, Lycklama A Nijeholt AA, et al. Chronic testicular pain as a symptom of pelvic floor dysfunction. J Urol 2010;183:177-81.

10.1016/j.juro.2009.08.147 [PubMed] [CrossRef] [Google Scholar]

19. Shoskes DA, Nickel JC, Kattan MW. Phenotypically directed multimodal therapy for chronic prostatitis/chronic pelvic pain syndrome: a prospective study using

UPOINT. Urology 2010;75:1249-53. 10.1016/j.urology.2010.01.021 [PubMed]

[CrossRef] [Google Scholar]

20. Farrell MR, Dugan SA, Levine LA. Physical therapy for chronic scrotal content pain with associated pelvic floor pain on digital rectal exam. Can J Urol 2016;23:8546-50.

[PubMed] [Google Scholar]

21. Tan WP, Tsambarlis PN, Levine LA. Microdenervation of the spermatic cord for postvasectomy pain syndrome. BJU Int 2018;121:667-73. 10.1111/bju.14125 [PubMed]

[CrossRef] [Google Scholar] 